University of Nebraska - Lincoln

DigitalCommons@University of Nebraska - Lincoln

$5-1990$

\title{
PYOVERDINE PRODUCTION BY PSEUDOMONAS AERUGINOSA EXPOSED TO METALS OR AN OXIDATIVE STRESS AGENT
}

\author{
Kim-Hien Thi Dao \\ University of Nebraska-Lincoln \\ Katherine E. Hamer \\ University of Nebraska-Lincoln \\ Christine L. Clark \\ University of Nebraska-Lincoln \\ Lawrence G. Harshman \\ University of Nebraska - Lincoln, Iharshman1@unl.edu
}

Follow this and additional works at: https://digitalcommons.unl.edu/bioscifacpub

Thi Dao, Kim-Hien; Hamer, Katherine E.; Clark, Christine L.; and Harshman, Lawrence G., "PYOVERDINE PRODUCTION BY PSEUDOMONAS AERUGINOSA EXPOSED TO METALS OR AN OXIDATIVE STRESS AGENT" (1990). Faculty Publications in the Biological Sciences. 274.

https://digitalcommons.unl.edu/bioscifacpub/274

This Article is brought to you for free and open access by the Papers in the Biological Sciences at DigitalCommons@University of Nebraska - Lincoln. It has been accepted for inclusion in Faculty Publications in the Biological Sciences by an authorized administrator of DigitalCommons@University of Nebraska - Lincoln. 


\title{
PYOVERDINE PRODUCTION BY PSEUDOMONAS AERUGINOSA EXPOSED TO METALS OR AN OXIDATIVE STRESS AGENT
}

\author{
Kim-Hien Thi Dao, Katherine E. Hamer, Christine L. Clark, and Lawrence G. Harshman ${ }^{1}$ \\ School of Biological Sciences, University of Nebraska-Lincoln, Lincoln, Nebraska 68588 USA
}

\begin{abstract}
Siderophores are low molecular mass compounds used by many microorganisms to scavenge dissolved iron, which is typically rare in environments. Pseudomonas aeruginosa (PAO1) was exposed to metals and methyl viologen in low-iron medium in order to monitor the abundance of a specific siderophore (pyoverdine) and monitor growth over time. In this study it was discovered that cadmium can stimulate pyoverdine abundance. Cadmium may interact with and sequester the Fur regulatory protein, which represses siderophore synthesis under conditions of iron sufficiency. Mercury did not stimulate pyoverdine production at concentrations that strongly inhibited bacterial growth, suggesting that pyoverdine production is not governed as part of a general stress response. Methyl viologen is an oxygen radical generator, and it was discovered that exposure to it decreased pyoverdine production. Decreased pyoverdine production may be a mechanism for reducing the iron potentiation of oxygen toxicity. It is hypothesized that factors stimulating production of siderophores may increase the susceptibility of microorganisms to oxidative damage. In general, our research suggests new opportunities for predicting ecotoxicological outcomes based on understanding of molecular mechanisms and the effect of xenobiotics or stress factors on fundamentally important microbial processes.
\end{abstract}

Key words: cadmium; mercury; methyl viologen; oxidative stress; Pseudomonas aeruginosa; siderophore.

\section{INTRODUCTION}

It is generally accepted that our ability to make predictions about the ecological effect of pollutants is very limited (Moriarty 1988). Studies of the effects of xenobiotics or other stressors on critical physiological processes in organisms may be especially useful for the purpose of improving our ability to predict the ecological impact of pollutants and stressors. The assumption is that physiological function is the logical point of integration for ecotoxicological studies. Specifically, the impact of pollutants, or environmental stress, on physiology is relevant to population growth, interaction between species, relative fitness, indicator gene expression, and the interaction between organisms and the environment. Physiological measures that are fundamentally important and connected with specific environmental states are attractive candidates for study.

Microbes are obvious candidates for such studies because they play an essential role in ecological communities. Moreover, they are capable of modifying the environment by their metabolic processes. This power is manifest in microenvironments, or on a larger scale, as in geochemical change. In addition, certain bacteria

Manuscript received 20 October 1997; accepted 27 April 1998; final version received 8 June 1998. For reprints of this Invited Feature, see footnote 1, page 429.

1 Address correspondence to this author. are model systems for the study of physiological processes. One example of the use of microbes for this approach is the study of phosphoenolpyruvate carboxylase regulation, which is motivated by the important role of this enzyme in the metabolism of (often limiting) dissolved organic matter in aquatic ecosystems by heterotrophic bacteria (Overbeck 1990). The effect of pollutants and stressors on critical aspects of microbial physiology is understudied with respect to the importance of microbes in the function of ecological communities. Such studies are valuable for the development of the field of ecotoxicology.

Iron acquisition is an essential physiological process for organisms. Iron is almost universally required for life, yet it is poorly soluble in the form of polyhydroxylated complexes at neutral pH (Neilands 1981). In some regions of the open ocean iron availability is the factor that limits phytoplankton population size (Kerr 1994). In order to meet this demand for iron, microbes, and other organisms use low molecular mass compounds, collectively called siderophores, to scavenge for iron (Guerinot 1994, Neilands 1995). Siderophores are important in relationship to the growth of aerobic and facultative anaerobic bacteria, fungal growth, plant growth, as well for their effects on the virulence of plant and human microbial diseases (Neilands 1981, Neilands and Leong 1986, Barton and Hemming 1993, Payne 1993). 
Pseudomonas aeruginosa is associated with aquatic and other habitats, and is also known as an opportunistic pathogen of humans. This species belongs to a group of microorganisms, the fluorescent pseudomonads, that are widely distributed across a range of habitats. Among the fluorescent pseudomonads, P. aeruginosa is the taxon best known in terms of genetics and physiology. $P$ aeruginosa produces two siderophores; pyoverdine is predominant, and has a much higher affinity for iron than the other siderophore, which is called pyochelin. The structure of pyoverdine is presented in Briskot et al. (1989). Pyoverdine is a peptide containing eight amino acids: $1 \mathrm{~L}$-arginine, $1 \mathrm{~L}$-lysine, 2 L-threonines, 2 L- $\delta \mathrm{N}$-hydroxyornithines, and $2 \mathrm{D}$ serines. The octahedral coordination of $\mathrm{Fe}(\mathrm{III})$ is accomplished by two $\delta \mathrm{N}$-hydroxyornithines and an (IS)5-amino-2,3-dihydro-8,9-dihydroxy-1 H-pyrimido[1,2-a]quinoline-1-carboxylic acid providing hydroxymate and catecholate donor oxygen atoms (Wendenbaum et al. 1983).

The present study addresses the question of whether exposure to xenobiotic stress factors results in a change in pyoverdine production by $P$. aeruginosa. Two of the metals employed in this study, cadmium and mercury, are significant environmental pollutants. In addition, experiments were conducted to determine if a nonmetal oxygen radical generator, methyl viologen (paraquat), could influence the production of pyoverdine. Paraquat is well established as a radical generator (Hassan and Fridovich 1979), and is used routinely to produce oxidative stress. Since iron is known to participate in the generation of oxygen radicals, exposure to this compound was motivated by the issue of iron management under conditions of oxidative stress.

\section{Materials AND Methods}

\section{Pyoverdine measurement and bacterial growth}

The fluorescent chromophore group of pyoverdine produces a characteristic pattern of excitation and emission (Meyer and Abdallah 1978). In the present study, a Perkin-Elmer (Oak Brook, Illinois) LS-5B luminescent spectrometer was used to qualitatively measure the abundance of pyoverdine by measuring fluorescence (excitation $405 \mathrm{~nm}$ and emission $450 \mathrm{~nm}$ ), which is functionally equivalent to an established method of pyoverdine abundance determination (Meyer and Abdallah 1978, Cox and Adams 1985, Visca et al. 1992). Low concentration of iron stimulates pyoverdine production, and higher concentrations, such as $10 \mu \mathrm{mol} / \mathrm{L}$ of supplemental iron, allows growth of $P$. aeruginosa with no siderophore production (Stintzi and Meyer 1994).

The $P$. aeruginosa strain used in this study was PAO1. Colonies were obtained by streak plating from $-80^{\circ} \mathrm{C} 15 \%$ glycerol storage onto Pseudomonas Agar $\mathrm{F}$ (PAF) medium. For aqueous culture, cells were grown in standard succinate medium (SSM). SSM is a low-iron medium that promotes siderophore production. Our experiments were designed to address the questions of whether added metals or an oxidative stress agent could stimulate or suppress the existing level of pyoverdine production in low iron medium. Growth in SSM was estimated by measurement of turbidity at an optical density of OD600 nm (Perkin-Elmer Spectrometer Lambda 3B). All solutions and media were prepared using double-distilled deionized water. Chemicals were from Sigma Chemical Company, St. Louis, Missouri unless otherwise stated.

\section{Metal assays}

In order to test the hypothesis that pyoverdine production by $P$. aeruginosa is influenced by the presence of heavy metals, dose response assays were conducted in which growth and pyoverdine production were monitored over time in samples taken from SSM culture. For these assays, PAO1 was inoculated from an overnight culture into $13 \times 100 \mathrm{~mm}$ borosilicate glass tubes (Fisher Scientific, Pittsburgh, Pennsylvania) containing SSM. All glassware used for cultures or assays was soaked in $0.1 \mathrm{~mol} / \mathrm{L} \mathrm{HCl}$ (Mallinckrodt Chemical, Paris, Kentucky) overnight to remove contaminating metals, followed by rinses with double-distilled water before autoclave sterilization. Overnight cultures were produced by inoculating a PAO1 colony from PAF medium into a $50-\mathrm{mL}$ long-neck culture flask with $25 \mathrm{~mL}$ $\mathrm{SSM}$. The growth period was $\sim 16 \mathrm{~h}$ at $25^{\circ} \mathrm{C}$ with moderate shaking at 150 cycles/min.

After overnight growth, cells were centrifuged for 5 min at $59 \times 10^{3} \mathrm{~m} / \mathrm{s}^{2}$ at room temperature. The pellet was resuspended in sterile $0.85 \% \mathrm{NaCl}$. The centrifugation and resuspension steps were repeated. The pellet from the second centrifugation was resuspended in $0.85 \% \mathrm{NaCl}$ to a standard final OD600 nm of 0.200 . A $20-\mu \mathrm{L}$ aliquot of this bacterial suspension was used as the inoculum into each $13 \times 100 \mathrm{~mm}$ assay tube containing $3 \mathrm{~mL}$ of SSM. The control tubes contained $3 \mathrm{~mL} \mathrm{SSM}$ with no added metals. The treatment tubes contained SSM with added metal chlorides $\left(\mathrm{CdCl}_{2}\right.$, $\mathrm{HgCl}_{2}, \mathrm{ZnCl}_{2}$ ). For cadmium and zinc, the experiments were conducted at final concentrations of $0.125,0.25$, 0.50 , and $1.00 \mathrm{mmol} / \mathrm{L}$. Solutions were prepared the day before inoculation of the assay tubes. The experiments with these metals were conducted four times. Mercury is highly toxic, and thus the concentrations employed (0.003-0.20 $\mu \mathrm{mol} / \mathrm{L})$ were much lower than the other metals. Replicate experiments using mercury were conducted with an extensive series of concentrations for the purpose of bracketing the transition to conditions that reduced growth of PAO1 in SSM.

Growth (OD600) and the qualitative estimate of pyoverdine production derived from the measure of fluorescence, excitation at $405 \mathrm{~nm}$ (ex405) and emission at $450 \mathrm{~nm}$ (em450), were recorded at 8-h intervals during a 64-h incubation period. The experiment was 


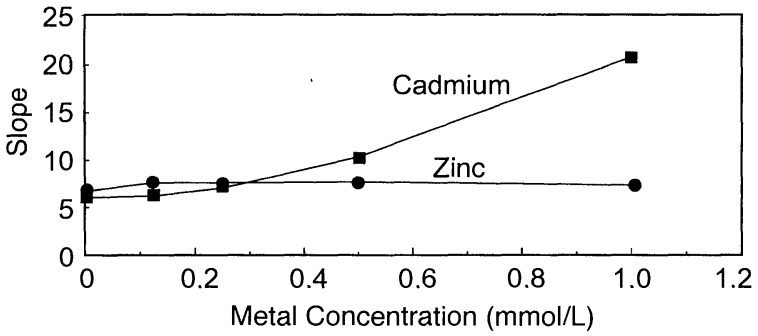

FIG. 1. Mean slope of pyoverdine produced (log excitation $405 \mathrm{~nm}$, emission $450 \mathrm{~nm} / \log$ OD600) as a function of added metal. Pseudomonas aeruginosa was continuously exposed to metals and sampled over time in standard succinate medium. The mean slope with no added metal was 6.85 .

designed so that there were replicate tubes for each assay time point, and only a single growth and pyoverdine reading was taken from any one tube. Assay tubes were not reused for successive readings. Luminometer measurements (ex405em450) were typically obtained from a 1:1000 dilution, but ranging from dilutions of $1: 100$ to $1: 10000$ depending on the level of fluorescence present in a sample.

Samples from SSM medium, and SSM medium with the high concentration of cadmium, were tested to determine how much of the characteristic pyoverdine fluorescence was quenched by addition of iron. The purpose was to estimate the proportion of residual fluorescent signal that could not be attributable to pyoverdine present in samples. The method was to dilute 64-h postinoculation samples to $\sim 1000$ luminometer units at ex405em450 and then add iron. Iron was added to a final concentration of $1.0 \mathrm{mmol} / \mathrm{L}$, which has been shown in this study to quench almost all of the characteristic fluorescent signal from semipurified pyoverdine.

\section{Semipurification of pyoverdine and metal quench tests}

Pyoverdine was semipurified to determine if the metals we employed in assays quenched the fluorescent signal used to estimate pyoverdine abundance. This was accomplished in a manner similar to that described in Meyer and Abdallah (1978). Overnight growth of PAO1 was obtained as described for the metals assay. Bacterial cells were pelleted, washed, and diluted to a final OD600 of 0.200 in $0.85 \% \mathrm{NaCl}$. A $1.0-\mathrm{mL}$ aliquot of the overnight culture was used as the inoculum into a 2-L Erlenmeyer flask containing $1.0 \mathrm{~L}$ of SSM. The culture in this flask was incubated at $25^{\circ} \mathrm{C}$ with moderate shaking for $72 \mathrm{~h}$. Thereafter, the cells were pelleted by centrifugation at $127.5 \times 10^{3} \mathrm{~m} / \mathrm{s}^{2}$ for $10 \mathrm{~min}$ at $25^{\circ} \mathrm{C}$. The supernatant was retained and concentrated by lyophilization. For lyophilization, $450 \mathrm{~mL}$ of culture supernatant was used to shell-coat the inside of bottles by rotation and freezing on the inside surface of a 1200$\mathrm{mL}$ lyophilization bottle using a dry ice-ethanol bath applied to the exterior of the bottle. Bottles were at- tached to a vacuum source and the contents freeze dried to a dry powder. The supernatant powder was resuspended in distilled water and solvent extracted as described by Meyer and Abdallah (1978).

Iron is known to quench pyoverdine fluorescence (Abdallah 1991). For the fluorescence quench assay, semipurified pyoverdine was measured before and after addition of different metals into solution. Semipurified pyoverdine was standardized with distilled water to $\sim 1000$ luminometer units (ex405em450) before addition of the test metals.

\section{Methyl viologen exposure}

In order to test the hypothesis that pyoverdine production is influenced by the presence of methyl viologen (paraquat), assays were conducted in which growth and pyoverdine production were monitored over time in SSM culture. Overnight cultures were grown, washed, and standardized as described for the metal assays. From a $100-\mathrm{mmol} / \mathrm{L}$ stock solution of methyl viologen, dilutions were made into SSM to final methyl viologen concentrations of $100 \mu \mathrm{mol} / \mathrm{L}$ and 500 $\mu \mathrm{mol} / \mathrm{L}$. Control tubes employed SSM without addition of methyl viologen. The tubes with SSM were inoculated with $20 \mu \mathrm{L}$ of standardized overnight culture of PAO1, as described for the metals assay. It is known that $P$. aeruginosa does not metabolize paraquat (Brown et al. 1995), and presumably metabolism of the compound does not play a role in these experiments. These assays were repeated a total of four times. The sampling intervals and total duration of incubation was longer than employed in the metal assays, because preliminary experiments indicated that it required longer to reach a reasonably consistent level of pyoverdine abundance.

\section{RESUlts}

\section{Metal assays}

The effects of cadmium and zinc on pyoverdine production are shown in Fig. 1. For each compound concentration, the average slope of the log of fluorescence (ex405em450) divided by log OD600 (pyoverdine production adjusted by growth) is presented for each concentration of a tested metal. The average slope for the controls, SSM without added metal, is 6.85. To show the effect of metals on the growth response, the average

TABLE 1. Mean slope of growth responses (log OD600) of Pseudomonas aeruginosa continuously exposed to different metals in standard succinate medium.

\begin{tabular}{lcccc}
\hline \hline \multirow{2}{*}{ Metal } & \multicolumn{4}{c}{ Concentration $(\mathrm{mmol} / \mathrm{L})$} \\
\cline { 2 - 5 } & 0.125 & 0.25 & 0.50 & 1.0 \\
\hline Cadmium & 0.018 & 0.017 & 0.013 & 0.005 \\
Iron & 0.027 & 0.027 & 0.023 & 0.014 \\
Zinc & 0.019 & 0.019 & 0.020 & 0.015 \\
\hline
\end{tabular}


TABLE 2. Mean slope of the growth response and pyoverdine production by Pseudomonas aeruginosa exposed to mercury concentrations in standard succinate medium for $64 \mathrm{~h}$.

\begin{tabular}{ccccccccc}
\hline \hline & \multicolumn{7}{c}{$\mathrm{HgCl}_{2}$ concentration $(\mu \mathrm{mol} / \mathrm{L})$} \\
\cline { 2 - 9 } Measure & 0 & 0.0031 & 0.0062 & 0.0125 & 0.025 & 0.050 & 0.100 & 0.200 \\
\hline $\begin{array}{l}\text { Mean slope of growth (log OD600) } \\
\begin{array}{l}\text { Mean slope of pyoverdine produc- } \\
\text { tion (log excitation 405 nm, emis- } \\
\text { sion 450 nm/log OD600) }\end{array}\end{array}$ & 0.286 & 0.325 & 0.340 & 0.332 & 0.362 & 0.305 & 0.252 & 0.212 \\
\hline
\end{tabular}

slope of the log of OD600 measurements is presented in Table 1.

In the present study, zinc did not have a substantial effect on growth or pyoverdine production, but cadmium was observed to affect both. The linear fit of the slope across a range of zinc concentrations is not significantly different from a slope of zero $(P=0.3950$, $\mathrm{df}=1)$. In contrast, cadmium acts to stimulate celldensity-adjusted pyoverdine production. In the case of cadmium, the linear fit of the slope of pyoverdine production is significant $(P<0.0001, \mathrm{df}=1)$, as is the fit to a quadratic slope $(P=0.0075, \mathrm{df}=1)$. The slope produced by $0.5 \mathrm{mmol} / \mathrm{L} \mathrm{Cd}$ is $140 \%$ greater than the control and $1 \mathrm{mmol} / \mathrm{L} \mathrm{Cd}$ is $300 \%$ greater than the control. The two higher concentrations of $\mathrm{Cd}$ stimulate pyoverdine production. This interpretation is supported by the statistical analysis, and inspection of the data suggests that the response is somewhat greater than loglinear (Fig. 1). There is evidence that the higher concentrations of cadmium are reducing the growth response (Table 1). Cadmium stimulates production of pyoverdine, but a question arises as to whether this is a nonspecific effect common to growth-inhibited (stressed) cells.

Table 2 presents the effect of mercury concentrations on growth and pyoverdine abundance. The mean slope values of $\log$ OD600 growth and log fluorescence at ex405em450 divided by log OD600 (pyoverdine production adjusted by growth) are shown for the 64-h growth period for each mercury concentration. In the concentration range from 0.003 to $0.05 \mu \mathrm{mol} / \mathrm{L}$, the effect of mercury relative to the control appears to be a slight increase in growth and slight decrease of the measure of cell-density-adjusted pyoverdine abundance. The higher concentrations of mercury decrease growth, as indicated by Table 2 and supported by the fact that the 64-h OD600 was at least 10-fold lower in the presence of the highest concentration of mercury compared to control growth (data not shown). The measure of pyoverdine adjusted by cell density decreases at higher mercury concentrations (Table 2). Consequently, it is apparent that there is no universal association between stress (reduction of growth) and an increase in pyoverdine production.

\section{Metal quench tests}

The quench effect of iron and other metals was tested on semipure pyoverdine. At a final concentration of 1.0 $\mathrm{mmol} / \mathrm{L} \mathrm{FeCl}_{3}$ the ex405em 450 measure of semipure pyoverdine was quenched $99.8 \%$. At the same concentration of iron using ex405em455, the fluorescence of semipure pyoverdine was quenched $99.7 \%$. At a final concentration of $0.1 \mathrm{mmol} / \mathrm{L} \mathrm{FeCl}{ }_{3}$ and ex $405 \mathrm{em} 450$, the fluorescence of semipure pyoverdine was quenched $96.9 \%$. At the same concentration of iron using ex $405 \mathrm{em} 455$, the fluorescence of semipure pyoverdine was quenched $97.3 \%$. At $0.1 \mathrm{mmol} / \mathrm{L}$ final concentration of iron, mercury quenched fluorescence $7.5 \%$, cadmium $2 \%$, and zinc $<0.5 \%$. Given the concentrations of mercury, cadmium, and zinc in solution after dilution of SSM cultures for fluorescence readings, it is unlikely that the metals significantly quenched fluorescence and thus impacted the measure of pyoverdine abundance in the experimental treatments. Moreover, there is no evidence that cadmium increases the fluorescent signal as an explanation for the increase in pyoverdine abundance when $P$. aeruginosa is exposed to cadmium in SSM.

Samples (64 $\mathrm{h}$ postinoculation) from the medium with the highest concentration of cadmium and control SSM were tested to determine how much of the characteristic pyoverdine fluorescence was quenched by addition of iron. At a final concentration of $1.0 \mathrm{mmol} / \mathrm{L}$ $\mathrm{FeCl}_{3}$ and fluorescence readings at ex405em450, control sample fluorescence was quenched $94.4 \%$ and the fluorescence from the SSM with the highest concentration of cadmium was quenched $95.6 \%$. At the same concentration of iron using ex $405 \mathrm{em} 455$, control sample was quenched $88.4 \%$ and the fluorescence from the SSM with the highest concentration of cadmium was quenched $93.5 \%$. Overall, the residual characteristic fluorescence that cannot be quenched by the addition of iron is small, suggesting that in this regard the measure of pyoverdine abundance is not subject to a bias sufficient to change the interpretation of the results. There is no indication that exposure to cadmium increases the proportion of this residual fluorescence.

\section{Methyl viologen assay}

Fig. 2 presents the pyoverdine production response of PAO1 cells to concentrations of methyl viologen. The mean variates are standardized to the control values to emphasize the pattern of response as a summary of four independent experiments. In each experiment, there was a significant reduction in the OD600-adjusted 


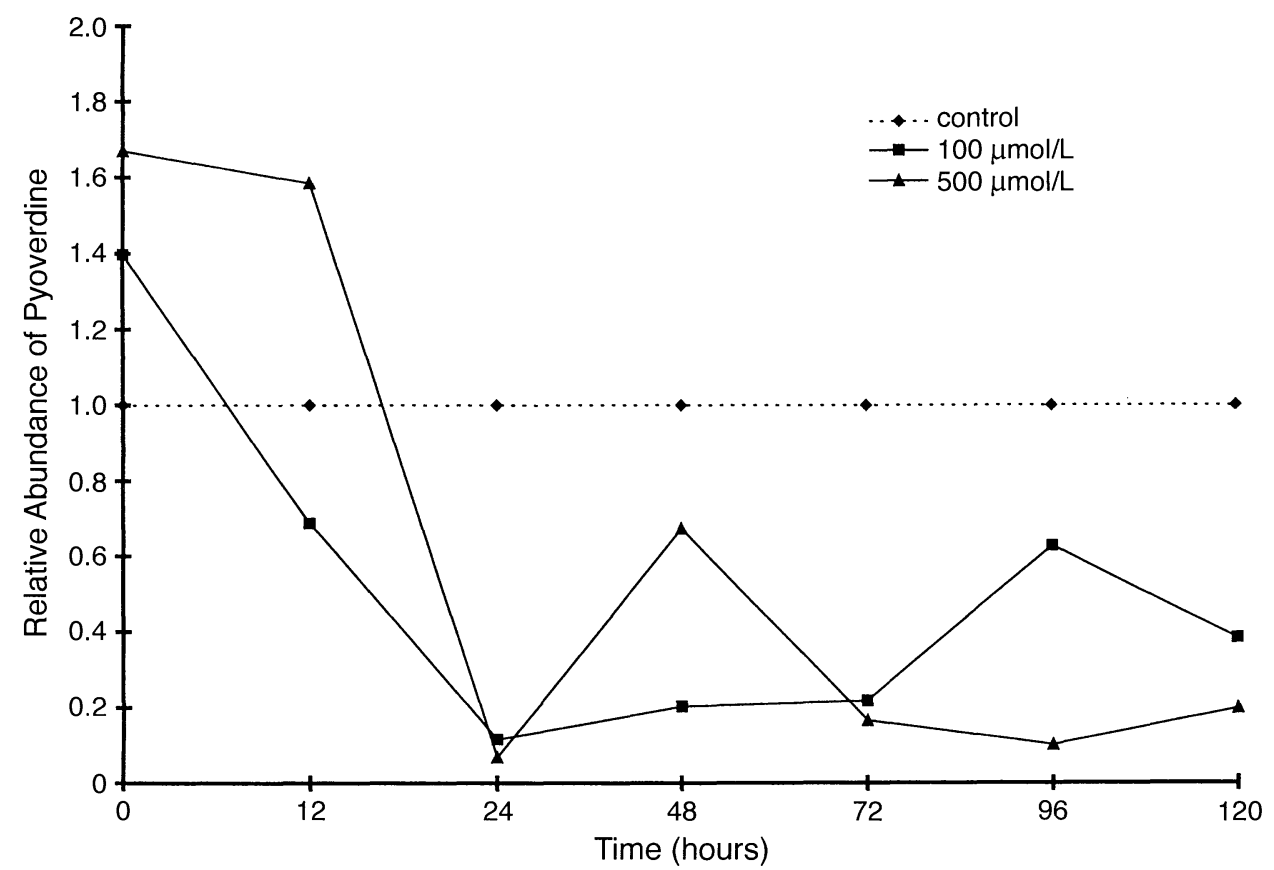

FIG. 2. Mean fluorescence (excitation $405 \mathrm{~nm}$, emission $450 \mathrm{~nm}$ ) adjusted by cell density (OD600) as a measure of pyoverdine produced by Pseudomonas aeruginosa continuously exposed to methyl viologen in standard succinate medium. The means of the treatment variates are presented relative to the control; the control with no methyl viologen added was set at 1.0 .

fluorescence. The level of support for significant treatment effects is $P=0.0004$ (experiment 1), $P=0.0495$ (experiment 2), $P=0.0001$ (experiment 3 ), and $P=$ 0.0039 (experiment 4 ). The data indicate that exposure to methyl viologen reduces pyoverdine production by PAO1 in SSM. Two earlier experiments (Hamer 1997) indicated that $100 \mu \mathrm{mol} / \mathrm{L}$ methyl viologen reduced fluorescence $\sim 50 \%$ relative to controls, compared to $60 \%$ in the experiments shown in Fig. 2, and 500 $\mu \mathrm{mol} / \mathrm{L}$ methyl viologen reduced fluorescence by $65 \%$, compared to $\sim 80 \%$ in the experiments shown in Fig. 2. From the earlier experiments it was observed that plated cells taken from the last time point of 500 $\mu \mathrm{mol} / \mathrm{L}$ methyl viologen cultures showed lower levels of pyoverdine production than control PAO1 when reintroduced into SSM aqueous medium (Hamer 1997). This observation suggests that exposure of cells to paraquat selects for genetic variants that produce less pyoverdine. Strong selection is consistent with the reduction in cell density observed at early time points after inoculation. The apparent temporal oscillatory pattern of the treatment pyoverdine measurements presented in Fig. 2 are not a significant feature of the data, given the standard errors of the 48- and 96-h time points. Overall, the reduction of pyoverdine production in response to oxidative stress is interpretable in terms of an adaptive response that reduces the iron-mediated potentiation of oxygen toxicity.

\section{DISCUSSION}

We are the first to document a stimulatory effect of cadmium on pyoverdine production, and also the first to document that exposure to methyl viologen can decrease siderophore production. In this discussion, we argue that these effects may be general, and we predict a negative synergistic effect of cadmium and paraquat based on the effect of cadmium on the molecular mechanisms that control siderophore synthesis and iron potentiation of oxidative stress.

Aspects of the mechanism of microbial control of siderophore production may be highly conserved. First described in Escherichia coli, siderophore synthesis is negatively regulated by iron interaction with Fur a repressor protein (Bagg and Neilands 1987). Specifically, $\mathrm{Fe}(\mathrm{II})$ is the ligand that interacts with Fur, which then binds to an operator suppressing expression of siderophore synthesis genes. Fur and fur gene homologs have been characterized from $P$. aeruginosa (Prince et al. 1991, 1993) and other taxa (Guerinot 1994). The DNA sequences required for iron regulation may also be conserved (Neilands 1995, Rombel et al. 1995). Moreover, in E. coli, an enterobactin (siderophore) iron complex is taken into the cell by a specific receptor protein (Raymond 1994). Similarly, after pyoverdine forms a complex with iron, membrane-bound receptors participate in ferripyoverdine uptake (Meyer et al. 1990, Poole et al. 1993). In general, when iron is lim- 
ited, gram-negative bacteria produce multiple outer membrane proteins as receptors which take up the ferrisiderophore complex, perhaps in a conserved manner by interaction with the TonB outer membrane protein (Guerinot 1994). As a result of mechanism conservation, data on the effects of xenobiotics and other environmental stressors on siderophore production in $P$. aeruginosa may extend to a range of microorganisms.

Various factors are known to influence siderophore production in fluorescent pseudomonads. As summarized by Meyer and Abdallah (1978), studies have shown that the nature of the carbon source in culture medium can influence production of pyoverdine-like pigment production. For $P$. aeruginosa, succinate was established as an optimal medium for production of this class of compounds (King et al. 1948) and this is likely to be a result of an iron requirement for the enzyme succino-dehydrogenase (Stinzi and Meyer 1994). Iron plays a pivotal role in controlling pyoverdine production by $P$. aeruginosa (Totter and Moseley 1953) and other microbes.

An additional layer of control of siderophore production has been documented in fluorescent pseudomonad taxa including $P$. aeruginosa (Leong et al. 1991, Cunliffe et al. 1995, Miyazaki et al. 1995). Totter and Moseley (1953) presented evidence that penicillin, but not streptomycin or chloromycetin, increased the production of pyoverdine-like pigment production in $P$. aeruginosa. Partially based on the work of Mergeay et al. (1978), Hofte et al. (1989) suggested that modulation of siderophore production was associated with a general stress response in various pseudomonads including $P$. aeruginosa. Antibiotics (ampicillin, tetracycline, and gentamycin) and metals (zinc and nickel) were implicated as stimulatory stressors. In contrast, the effect of mercury in our study does not support the hypothesis of increased pyoverdine production as a general stress response.

In our study, cadmium was found to stimulate pyoverdine abundance but zinc had no effect. The earlier assertion that cadmium inhibited pyoverdine production was based on a description of an interaction with cobalt resistance, but no data were presented to document this assertion (Mergeay et al. 1978). Our work, documenting a dose-dependent stimulation of pyoverdine abundance, supports the hypothesis that cadmium has a stimulating effect on pyoverdine production. Work on E. coli suggests that our results are correct. Specifically, in E. coli it has been shown that cadmium interacts with Fur protein thiols with high avidity, but the liganded repressor has reduced affinity for regulatory DNA sequences as determine by in vitro footprinting assays (Coy and Neilands 1991).

We did not observe an effect of zinc on pyoverdine production as might be expected by the results of Hofte et al. (1993, 1994). The activation by zinc (Hofte et al. $1993)$ is associated with a site-specific recombinase gene (Hofte et al. 1994). Their studies investigated activation of siderophore expression in the presence of relatively high iron concentration, whereas our study was an investigation of the possible stimulatory effect of zinc when iron concentration is sufficiently low to allow pyoverdine production. Noteworthy in this regard is the data showing that $100 \mu \mathrm{mol} / \mathrm{L}$ (or less) of zinc in low iron medium did not stimulate pyoverdine production by $P$. aeruginosa PAO1 (Visca et al. 1992). Alternatively, there may be differences between the 7NSK2 strain used by Hofte et al. (1993, 1994) and PAO1 used in our study and the work by Visca et al. (1992). Finally, differential outcomes may be derived from the use of plate assays (Hofte et al. 1993, 1994) vs. the aqueous culture assays used in the present study. In support of this possibility, a recombinase mutation continued to synthesize pyoverdine in LB broth supplemented with zinc, but not on an LB plate supplemented with zinc (Hofte et al. 1994).

Physiological measures can be used to detect and integrate the biological impact of stress factors. In spite of the intuitive appeal of physiological indicators, Bayne et al. (1979) has noted that general measures such as growth tend to obscure the identity of specific stress factors. Pyoverdine production is a specific physiological response that may provide some evidence about the nature of the stressor. However, it is increasingly apparent that the $P$. aeruginosa Fur regulatory system is complex, involving an array of coordinately regulated genes as well as various sigma factors and other global regulators (Tardat and Touati 1993, Cunliffe et al. 1995, Ochsner and Vasil 1996). Consequently, it is not clear to what degree a pyoverdine response could provide evidence about the specific identity of a stressor.

Iron potentiates oxygen toxicity, and virtually all organisms are expected to have evolved mechanisms for the purpose of iron management (Kaplan and O'Halloran 1996). Our study suggests that methyl viologen (paraquat) may act to suppress pyoverdine production. During $P$. aeruginosa culture, the reduction of oxygen in culture during stationary phase is associated with a burst of pyoverdine production under low iron conditions (Cox 1986) and an increase in potential in the cell (Hassan and Moody 1987). Methyl viologen is known to induce catalase and superoxide dismutase activities, presumably as oxygen stress protection mechanisms (Hassett et al. 1993, Brown et al. 1995). Fur mutations can have altered catalase and superoxide dismutase activities, and are defective in pyoverdine synthesis (Hassett et al. 1996). The sensitivity of these mutants to hydrogen peroxide and paraquat was greater than wild-type, and the suggestion was made that reduction in pyoverdine synthesis could be a contributing factor (Hassett et al. 1996). Perhaps in contrast, our results indicate that reduced pyoverdine production may be a consequence of exposure to paraquat, pre- 
sumably to reduce the amount of iron assimilation and thereby reduce the potentiation of its toxic effects. Given the complexity of iron and oxygen regulation of gene expression in pseudomonads (Tardat and Touati 1993), it is plausible that under some circumstances pyoverdine expression could be decoupled from coordinate upregulation with catalase and superoxide dismutase activities.

The ecological consequences of maladaptive regulation of siderophore production is significant because iron management is a universal problem for organisms. For example, constitutive siderophore expression in $E$. coli results in increased sensitivity to near UV irradiation (Hoerter et al. 1996). In general, DNA-damaging oxygen radicals challenge the viability of microorganisms (Linn and Imlay 1987, Arrage et al. 1993).

Our research suggests new opportunities for predicting ecotoxicological outcomes based on the interaction of xenobiotics with molecular mechanisms that regulate key microbial processes. Specifically, based on the results of this study we are able to propose a hypothesis that relates pyoverdine production, cellular iron accumulation, and oxygen toxicity. The hypothesis could have general implications given the conserved mechanisms underlying control of siderophore synthesis. Importantly, we can make the detailed prediction that the effect of cadmium in stimulating pyoverdine production will predominate over the effect of paraquat in reducing pyoverdine production when $P$. aeruginosa is exposed simultaneously to both xenobiotics. In this scenario, the stimulatory effect of cadmium would be predominant because a diffusable factor, cadmium, is expected to reduce the functional availability of the Fur protein, which serves to repress gene expression for pyoverdine synthesis. Consequently when both cadmium and methyl viologen are present, the cell is hypothesized to take up an excess of iron by the vehicle of pyoverdine overproduction under conditions of oxidative stress due to the presence of methyl viologen. In this manner, the cell would amplify oxidative stress because iron potentiates oxygen toxicity. In general, we are arguing that a future avenue of ecotoxicological research should be to conduct mechanism-driven studies of synergistic effects of xenobiotics, or other stressors, based on fundamentally important features of microbial physiological processes.

\section{ACKNOWLEDGMENTS}

This research was supported by the metallobiochemistry program at the University of Nebraska-Lincoln and by NSFEPSCOR project 92-555225. We thank Professor Nielands for sharing his experience and insight, Ming-Hoi Wu for capable assistance, and Tony Joern for editorial contributions. In addition, we would like to thank Ronald Gorman and Kathie Helzer for diligent help in collecting data.

\section{LiterATURE Cited}

Abdallah, M. A. 1991. Pyoverdines and pseudobactins. Pages $139-153$ in G. Winkelman, editor. CRC handbook of microbial iron chelates. CRC Press, Ann Arbor, Michigan, USA.

Arrage, A. A., T. J. Phelps, R. E. Benoit, and D. C. White. 1993. Survival of subsurface microorganisms exposed to UV radiation and hydrogen peroxide. Applied Environmental Microbiology 59:3545-3550.

Bagg, A., and J. B. Neilands. 1987. Ferric uptake regulation protein acts as a repressor, employing iron(II) as a cofactor to bind the operator of an iron transport operon in Escherichia coli. Biochemistry 26:5471-5477.

Barton, L. L., and B. C. Hemming. 1993. Iron chelation in plants and soil microorganisms. Academic Press, New York, New York, USA.

Bayne, B. L., D. A. Brown, K. Burns, D. R. Dixon, A. Ivanovici, D. R. Livingstone, D. M. Lowe, M. N. Moore, A. R. D. Stebbing, and J. Widdows. 1979. An experimental approach to the determinants of biological water quality. Philosophical Transactions of the Royal Society of London B 286:563-581.

Briskot, G., K. Taraz, and H. Budikiewicz. 1989. Pyoverdintype siderophores from Pseudomonas aeruginosa. Liebigs Annalen Der Chemie 4:375-384.

Brown, S. M., M. L. Howell, M. L. Vasil, A. J. Anderson, and D. J. Hassett. 1995. Cloning and characterization of the katb gene of Pseudomonas aeruginosa encoding, a hydrogen peroxide-inducible catalase: purification of KatB, cellular localization, and demonstration that it is essential for optimal resistance to hydrogen peroxide. Journal of Bacteriology 177:6536-6544.

Cox, C. D. 1986. Relationship between oxygen and siderophore synthesis in Pseudomonas aeruginosa. Current Microbiology 14:19-23.

Cox, C. D., and P. Adams. 1985. Siderophore activity of pyoverdine for Pseudomona aeruginosa. Journal of Bacteriology 137:357-364.

Coy, M., and J. B. Neilands. 1991. Structural dynamics and functional domains of the Fur protein. Biochemistry 30: 8201-8210.

Cunliffe, H. E., T. R. Merriman, and I. L. Lamont. 1995. Cloning and characterization of $p v d S$, a gene required for pyoverdine synthesis in Pseudomonas aeruginosa: $\mathrm{PvdS}$ is probably an alternative sigma factor. Journal of Bacteriology 177:2744-2750.

Guerinot, M. L. 1994. Microbial iron transport. Annual Review of Microbiology 48:743-772.

Hamer, K. 1997. Production of pyoverdin by Pseudomonas aeruginosa after exposure to stress conditions. Thesis. University of Nebraska-Lincoln, Lincoln, Nebraska, USA.

Hassan, H. M., and I. Fridovich. 1979. Paraquat and Escherichia coli: mechanism of production of extracellular superoxide radical. Journal of Biological Chemistry 253: 10846-10852.

Hassan, H. M. and C. S. Moody. 1987. Regulation of manganese-containing superoxide dismutase in Escherichia coli: anaerobic induction by nitrate. Journal of Biological Chemistry 262:17173-17177.

Hassett, D. J., P. A. Sokol, M. L. Howell, J. F. Ma, H. T. Schweizer, U. Ochsner, and M. L. Vasil. 1996. Ferric uptake regulators (Fur) mutants of Pseudomonas aeruginosa demonstrate defective siderophore-mediated iron uptake, altered aerobic growth, decreased superoxide dismutase and catalase activities. Journal of Bacteriology 178:39964003.

Hassett, D. J., W. A. Woodruff, D. J. Wozniak, M. L. Vasil, M. S. Cohen, and D. E. Ohman. 1993. Cloning and characterization of the Pseudomonas aeruginosa sodA and $\operatorname{sodB}$ genes encoding manganese- and iron-cofactored superoxide dismutase: demonstration of increased manganese superoxide dismutase activity in alginate-producing bacteria. Journal of Bacteriology 175:7658-7665. 
Hoerter, J., A. Pierce, C. Troupe, J. Epperson, and A. Eisenstark. 1996. The role of enterobactin and intracellular iron in cell lethality during near-UV irradiation in E. coli. Photochemistry and Photobiology 64:537-541.

Hofte, M., S. Buysens, N. Koedam, and P. Cornelis. 1993. Zinc affects siderophore-mediated high affinity iron uptake systems in the rhizoshere Pseudomonas aeruginosa 7NSK2. Biological Metals 6:85-91.

Hofte, M., Q. Dong, S. Kourambas, V. Krishnapillai, D. Sherrat, and M. Mergeay. 1994. The sss gene product, which affects pyoverdine production in Pseudomonas aeruginosa $7 \mathrm{NSK} 2$, is a site-specific recombinase. Molecular Microbiology 14:1011-1020.

Hofte, M., L. Mergeay, L. Diels, and W. Verstraete. 1989. Influence of stress factors on siderophore production by fluorescent Pseudomonads. Societe Belge de Biochimie, Gent. 4:B96.

Kaplan, J., and T. V. O'Halloran. 1996. Iron metabolism in eukaryotes: Mars and Venus at it again. Science 271:15101512.

Kerr, R. A. 1994. Iron fertilization: a tonic, but no cure for the greenhouse. Science 263:1089-1090.

King, J. V., J. J. R. Campbell, and B. A. Eagles. 1948. The mineral requirements for fluorescein production. Canadian Journal of Research 26C:514-519.

Leong, J., W. Bitter, M. Koster, V. Venturi, and P. J. Weisbeek. 1991. Molecular analysis of iron transport in plant growthpromoting Pseudomonas putida WCS358. Biological Metals 4:36-40.

Linn, S., and J. A. Imlay. 1987. Toxicity, mutagenesis and stress responses induced in $E$. coli by hydrogen peroxide. Journal of Cell Science, Supplement 6:289-301.

Mergeay, M., J. Gerits, and C. Houra. 1978. Facteur transmissible de la resistance au cobalt chez un Pseudomonas du type hydrogenomonas. C. R. Soc. Biol. 172:575-579.

Meyer, J. M., and M. A. Abdallah. 1978. The fluorescent pigment of Pseudomonas fluorescens: biosynthesis, purification, and physiochemical properties. Journal of General Microbiology 107:319-328.

Meyer, J. M., D. Hohnadel, A. Khan, and P. Cornelius. 1990. Pyoverdine-facilitated iron uptake in Pseudomonas aeruginosa: immunological characterization of the ferripyoverdine receptor. Molecular Microbiology 4:1401-1405.

Miyazaki, H., H. Kato, T. Nakazawa, and M. Tsuda. 1995. A positive regulatory gene, $p v d S$, for expression of pyoverdine biosynthetic genes in Pseudomonas aeruginosa. Molecular and General Genetics 248:17-24.

Moriarty, F. 1988. Ecotoxicology, the study of pollutants in ecosystems. Academic Press, London, UK.

Neilands, J. B. 1981. Microbial iron compounds. Annual Review of Biochemistry 50:715-73.

1995. Siderophores: structure and function of mi- crobial iron transport compounds. Journal of Biological Chemistry 270:26723-26726.

Neilands, J. B., and S. A. Leong. 1986. Siderophores in relationship to plant growth and disease. Annual Review of Plant Physiology 37:187-208.

Ochsner, U. A., and M. L. Vasil. 1996. Gene repression by the ferric uptake regulator in Pseudomonas aeruginosa: cycle selection by iron-regulated genes. Proceedings of the National Academy of Science (USA) 93:4409-4414.

Overbeck, J. 1990. Aspects of aquatic microbial carbon metabolism: regulation of phosphoenolpyruvate carboxylase. Pages 70-95 in J. Overbeck and R. Chrost, editors. Aquatic microbial ecology. Springer-Verlag, Berlin, Germany.

Payne, S. M. 1993. Iron acquisition in microbial pathogenesis. Trends in Microbiology 1:66-69.

Poole, K., S. Neshat, K. Krebes, and D. E. Heinrichs. 1993. Cloning and nucleotide sequence analysis of the ferripyoverdine receptor gene $f p v A$ of $P$ seudomonas aeruginosa. Journal of Bacteriology 175:4597-4604.

Prince, R. W., C. D. Cox, and M. L. Vasil. 1993. Coordinate regulation of siderophore and exotoxin A production: molecular cloning and sequencing of the Pseudomonas aeruginosa fur gene. Journal of Bacteriology 175:2589-2598.

Prince, R. W., D. G. Storey, A. I. Vasil, and M. L. Vasil. 1991. Regulation of toxA and regA by the Escherichia coli fur gene and identification of a Fur homologue in Pseudomonas aeruginosa PA103 and PAO1. Molecular Microbiology 5:2823-2831.

Raymond, K. N. 1994. Recognition and transport of natural and synthetic siderophores by microbes. Pure and Applied Chemistry 66:773-781.

Rombel, I. T., B. J. McMorran, and I. L. Lamont. 1995. Identification of a DNA sequence motif required for expression of iron-regulated genes in pseudomonads. Molecular and General Genetics 246:519-528.

Stintzi, A., and J.-M. Meyer. 1994. Search for siderophores in micro-organisms. Microbes for Better Living 35:169176.

Tardat, B., and D. Touati. 1993. Iron and oxygen regulation of Escherichia coli MnSOD expression: competition between the global regulators Fur and ArcA for binding to DNA. Molecular Microbiology 9:53-63.

Totter, J. R., and F. T. Moseley. 1953. Influences of the concentration of iron on the production of fluorescein by Pseudomonas aeruginosa. Journal of Bacteriology 65:45-47.

Visca, P., G. Colotti, L. Serino, D. Verzili, N. Orsi, and E. Chiancone. 1992. Metal regulation of siderophore synthesis in Pseudomonas aeruginosa and functional effects of siderophore-metal complexes. Applied and Environmental Microbiology 58:2886-2893.

Wendenbaum, S., P. Demange, A. Dell, J. M. Meyer, and M. A. Abdallah. 1983. The structure of pyoverdine $\mathrm{Pa}$, the siderophore of Pseudomonas aeruginosa. Tetrahedron Letters 24:4877-4880. 